\title{
Defects in homologous recombination repair genes are associated with good prognosis and clinical sensitivity to DNA-damaging agents in pancreatic cancer: A case report
}

\author{
AMIR SONNENBLICK, AVIAD ZICK, MYRIAM MAOZ, SHERRI COHEN, \\ LUNA KADOURI, TAMAR PERETZ and AYALA HUBERT
}

Sharett Institute of Oncology, Hadassah-Hebrew University Medical Center, Ein Kerem, Jerusalem 91120, Israel

Received September 4, 2017; Accepted December 7, 2017

DOI: $10.3892 /$ mco.2018.1588

\begin{abstract}
Tumor genome sequencing is important for increasing our understanding of the development of cancer, which may be affected by different therapies. In the present study, genomic evolution was investigated in a patient with stage IV pancreatic cancer bearing a germline breast cancer 2 (BRCA2) mutation. The patient received cisplatin, a DNA cross-linking agent, which led to a long-lasting complete response. Eventually the patient developed brain metastasis, suggesting the acquisition of resistance to cisplatin. He subsequently underwent brain lesion resection, radiofrequency ablation and chemotherapy, again resulting in long-lasting response. Samples of blood, pancreatic tumor tissue and brain metastases were collected and the extracted DNA was sequenced. The pancreatic and brain lesions, when compared with the blood samples, exhibited mutations in the BRCAl and checkpoint kinase 2 genes, in addition to the germline $B R C A 2$ mutation. The brain lesion, when compared with the primary tumor, harbored no additional mutations or copy-number variations. These findings suggest that the isolated relapse in the brain was due to pharmacological sanctuary rather than genomic alterations. It may be suggested that the presence of defects in the homologous recombination repair pathways are associated with a good prognosis and clinical sensitivity to agents that damage the DNA in pancreatic cancer.
\end{abstract}

\section{Introduction}

Pancreatic cancer is a deadly disease and it is usually diagnosed at an advanced stage. Chemotherapy plays a key role

Correspondence to: $\mathrm{Dr}$ Amir Sonnenblick, Sharett Institute of Oncology, Hadassah-Hebrew University Medical Center, Ein Kerem, P.O. Box 12000, Jerusalem 91120, Israel

E-mail: amirsonn@gmail.com

Key words: breast cancer 1, breast cancer 2, checkpoint kinase 2, pancreatic adenocarcinoma, cisplatin, DNA damage, genome sequencing, synthetic lethality, chemotherapy, pharmacological sanctuary in the treatment of pancreatic cancer, often producing a temporary clinical benefit $(1,2)$. Genomic aberrations in breast cancer $1(B R C A 1)$ and $B R C A 2$ genes, which are components of a common DNA repair pathway, are inherited in an autosomal dominant pattern $(3,4)$. A number of studies have demonstrated that $B R C A$ mutations, particularly those in $B R C A 2$, increase the risk of developing pancreatic adenocarcinoma (5). It has been suggested that DNA-damaging agents, such as platinum salts or poly(ADP-ribose) polymerase (PARP) inhibitors, may be used in patients with pancreatic cancer carrying $B R C A$ mutations $(6,7)$. In the present study, genomic evolution was investigated in a patient with stage IV pancreatic cancer bearing a germline BRCA2 mutation.

\section{Case report}

A 60-year-old male patient carrying a known deleterious BRCA2 mutation (1153insT) presented with locally advanced pancreatic cancer. The patient underwent surgery, and the subsequent pathological analysis revealed pancreatic adenocarcinoma. Two months later, prior to commencing adjuvant chemotherapy, the patient developed liver metastasis. He received a cisplatin-based regimen and rapidly achieved a complete response that lasted for 18 months. When relapse occurred in the liver, the patient resumed the same protocol, achieving a partial response for an additional 20 months. At that point, disease progression was detected in the brain. The patient received multidisciplinary treatment that included resection of one lesion and stereotactic radiosurgery (SRS) for the second lesion. Subsequently, he received irinotecan and bevacizumab, which resulted in a response that lasted for 7 months.

The patient provided written informed consent, in accordance with the Hadassah Institutional Review Board-approved protocol.

DNA isolation. Formalin-fixed, paraffin-embedded (FFPE) tumor tissues were assessed by a board-certified pathologist. The regions of tumor tissue were marked and the DNA was extracted using a QIAamp DNA FFPE Tissue kit (Qiagen, Solana Beach, CA, USA). DNA was extracted from the blood using a DNeasy Blood and Tissue kit (Qiagen), according to the manufacturer's instructions. 
Table I. Somatic mutations detected in primary tumor and metastases.

Allele frequency $(\%)$

\begin{tabular}{lllcc} 
Mutation & \multicolumn{1}{c}{ RNA } & \multicolumn{1}{c}{ Protein } & Pancreas (primary) & Brain (metastasis) \\
\hline BRCA1 & NM_007294.3:c.4535G>T & NP_009225.1:p.Ser1512Ile & 47 & 57 \\
P53 & c.831T>ANM_001126112.2 & NP_000537.3:p.C277* & 33 & 46 \\
K-RAS & NM_004985.4:c.35G>C & NP_004976.2:p.Gly12Asp & 44 & 32 \\
CHEK2 & NM_001005735.1:c.1399T>C & NP_001005735.1:p.Tyr467His & 50 & 57
\end{tabular}

BRCA1, breast cancer 1; CHEK2, checkpoint kinase 2.

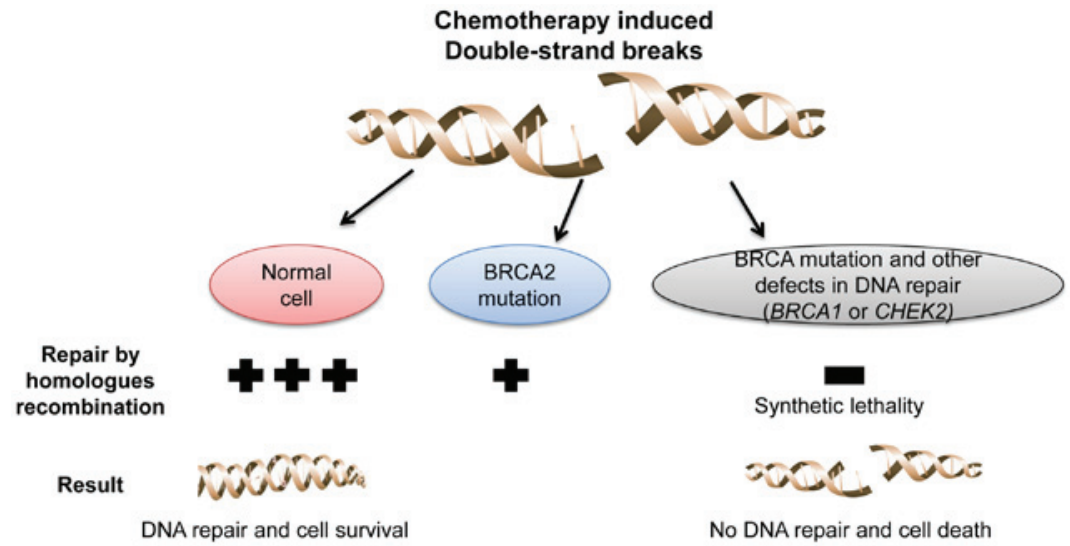

Figure 1. Loss-of-function mutations of breast cancer 1 (BRCA1), BRCA2 and checkpoint kinase 2 (CHEK2) may sensitize cancer cells to DNA-damaging agents to a greater extent, compared with loss of function of only one.

Massive parallel sequencing. The clinical samples were screened for mutations in 50 cancer-associated genes using an Ion AmpliSeq ${ }^{\mathrm{TM}}$ Cancer Hotspot Panel v2, and a panel spanning the coding sequences of an additional 22 cancer-associated genes. DNA extraction and sequencing were performed as previously described (8).

The blood, primary tumor tissue (prior to any treatment) and the brain metastatic tissue were examined using a targeted deep sequencing assay with a mean 1,320-fold coverage. The known deleterious BRCA2 mutation (1153insT) was identified in all three samples, while a BRCAl mutation (NM_007294.3:c.4535G >T; NP_009225.1:p.Ser1512Ile; rs1800744) and a checkpoint kinase 2 (CHEK2) mutation (NM_001005735.1:c.1399T>C; NP_001005735.1:p. Tyr467His) were identified only in the pancreatic tumor tissue and brain metastasis. The pancreatic and brain samples shared other somatic genetic aberrations (Table I), but no significant mutational differences were detected between the two. This suggests that the isolated relapse in the brain was due to pharmacological sanctuary rather than further genomic alternations. The patient survived $\sim 7$ years with metastatic disease until succumbing to his illness.

\section{Discussion}

In order to identify biomarkers for response and resistance to platinum-based therapy in exceptional responder, parallel genomic molecular characterization of the primary and metastatic tumors was conducted. Genetic sequencing indicated that selective therapeutic pressure did not lead to any significant genomic alternation in the brain metastasis, which suggests that the isolated relapse in the brain was instead due to pharmacological sanctuary at this site. This hypothesis was confirmed by the clinical observation that the patient also responded well to SRS and irinotecan administered to treat the brain recurrence, suggesting that the therapeutic sensitivity to DNA-damaging agents was retained.

Moreover, a BRCAl gene mutation was identified in both the primary tumor tissue and the brain metastasis, in addition to the germline BRCA2 mutation. Such dual BRCA1/2 loss of function may be the cause of the exceptional clinical sensitivity to DNA-damaging agents throughout the treatment of this patient.

In light of the role of $B R C A$ in DNA repair, it is suggested that $B R C A 1$ or $B R C A 2$ mutations result in increased sensitivity to DNA-damaging agents $(9,10)$. Indeed, mounting evidence suggests a better response to PARP inhibitors or cisplatin in $B R C A$-associated malignancies (11-16). However, over time, $B R C A$-deficient tumors become resistant and disease progression may occur.

The 'synthetic lethality' concept has provided new opportunities for drug development (17). For example, in cancer cells with loss of function of $B R C A$, treatment with PARP inhibitors leads to an accumulation of single-strand breaks that subsequently develop into double-strand breaks, which cannot be fixed by homologous recombination $(18,19)$. 
A mutation in the CHEK2 gene in the primary tumor and brain metastatic tissues was also detected. CHEK2 is an important regulator of cellular response to DNA damage, and has been identified as tumor-suppressor gene in various human malignancies $(20,21)$. This CHEK2 mutation may also contribute to the defects in DNA repair mechanisms in the described tumor.

To conclude, the findings of the present study suggest that loss of BRCA1, BRCA2 and CHEK2 function may result in greater sensitivity of cancer cells to DNA-damaging agents compared with the loss of function of only one of these genes (Fig. 1). If such a strategy becomes pharmacologically applicable, it may represent a novel synthetic effective approach to the treatment of pancreatic cancer, as well as other malignancies.

\section{Acknowledgements}

We would like to thank Tamar H. for her assistance.

\section{Funding}

AS is supported by a Clinical Research Career Development Award from the Israel Cancer Research Fund grants (16-116-CRCDA) and from the Israeli cancer research association (2017-0140).

\section{Availability of data and materials}

Not applicable

\section{Authors' contributions}

AS, AZ, TP and AH conceived of the study and participated in its design and coordination, analyzed and interpreted the data and wrote the manuscript. MM and SC performed technical work.

\section{Ethics approval and consent to participate}

The patient provided written informed consent for the genetic research studies performed in accordance with protocols approved by the Institutional Review Board.

\section{Consent for publication}

Not applicable.

\section{Competing interests}

The authors declare that they have no competing interests.

\section{References}

1. Gresham GK, Wells GA, Gill S, Cameron C and Jonker DJ: Chemotherapy regimens for advanced pancreatic cancer: A systematic review and network meta-analysis. BMC Cancer 14: 471, 2014.

2. Heinemann V, Haas M and Boeck S: Systemic treatment of advanced pancreatic cancer. Cancer Treat Rev 38: 843-853, 2012.

3. Roy R, Chun J and Powell SN: BRCA1 and BRCA2: Different roles in a common pathway of genome protection. Nat Rev Cancer 12: 68-78, 2011.
4. Mavaddat N, Peock S, Frost D, Ellis S, Platte R, Fineberg E, Evans DG, Izatt L, Eeles RA, Adlard J, et al: Cancer risks for BRCA1 and BRCA2 mutation carriers: Results from prospective analysis of EMBRACE. J Natl Cancer Inst 105: 812-822, 2013.

5. Hahn SA, Greenhalf B, Ellis I, Sina-Frey M, Rieder H, Korte B, Gerdes B, Kress R, Ziegler A, Raeburn JA, et al: BRCA2 germline mutations in familial pancreatic carcinoma. J Natl Cancer Inst 95: 214-221, 2003.

6. Sonnenblick A, Kadouri L, Appelbaum L, Peretz T, Sagi M, Goldberg Y and Hubert A: Complete remission, in BRCA2 mutation carrier with metastatic pancreatic adenocarcinoma, treated with cisplatin based therapy. Cancer Biol Ther 12: 165-168, 2011.

7. Lowery MA, Kelsen DP, Stadler ZK, Yu KH, Janjigian YY, Ludwig E, D'Adamo DR, Salo-Mullen E, Robson ME, Allen PJ, et al: An emerging entity: Pancreatic adenocarcinoma associated with a known BRCA mutation: Clinical descriptors, treatment implications and future directions. Oncologist 16: 1397-1402, 2011

8. Zick A, Peretz T, Lotem M, Hubert A, Katz D, Temper M, Rottenberg Y, Uziely B, Nechushtan H, Meirovitz A, et al: Treatment inferred from mutations identified using massive parallel sequencing leads to clinical benefit in some heavily pretreated cancer patients. Medicine (Baltimore) 96: e6931, 2017.

9. Muggia F, Safra T and Dubeau L: BRCA genes: Lessons learned from experimental and clinical cancer. Ann Oncol 22 (Suppl 1): i7-i10, 2011

10. Sonnenblick A, de Azambuja E, Azim HA Jr and Piccart M: An update on PARP inhibitors-moving to the adjuvant setting. Nat Rev Clin Oncol 12: 27-41, 2015.

11. Silver DP, Richardson AL, Eklund AC, Wang ZC, Szallasi Z, Li Q, Juul N, Leong CO, Calogrias D, Buraimoh A, et al: Efficacy of neoadjuvant cisplatin in triple-negative breast cancer. J Clin Oncol 28: 1145-1153, 2010.

12. Fong PC, Boss DS, Yap TA, Tutt A, Wu P, Mergui-Roelvink M, Mortimer P, Swaisland H, Lau A, O'Connor MJ, et al: Inhibition of Poly(ADP-Ribose) polymerase in tumors from BRCA mutation carriers. N Engl J Med 361: 123-134, 2009

13. Ledermann J, Harter P, Gourley C, Friedlander M, Vergote I, Rustin G, Scott CL, Meier W, Shapira-Frommer R, Safra T, et al: Olaparib maintenance therapy in patients with platinum-sensitive relapsed serous ovarian cancer: A preplanned retrospective analysis of outcomes by BRCA status in a randomised phase 2 trial. Lancet Oncol 15: 852-861, 2014.

14. Kwa M, Edwards S, Downey A, Reich E, Wallach R, Curtin J and Muggia F: Ovarian cancer in BRCA mutation carriers: Improved outcome after intraperitoneal (IP) cisplatin. Ann Surg Oncol 21: $1468-1473,2014$

15. Golan T, Kanji ZS, Epelbaum R, Devaud N, Dagan E, Holter S, Aderka D, Paluch-Shimon S, Kaufman B, Gershoni-Baruch R, et al: Overall survival and clinical characteristics of pancreatic cancer in BRCA mutation carriers. Br J Cancer 111: 1132-1138, 2014

16. Fong PC, Yap TA, Boss DS, Carden CP, Mergui-Roelvink M, Gourley C, De Greve J, Lubinski J, Shanley S, Messiou C, et al: Poly(ADP)-ribose polymerase inhibition: frequent durable responses in BRCA carrier ovarian cancer correlating with platinum-free interval. J Clin Oncol 28: 2512-2519, 2010.

17. Kaelin WG Jr: The concept of synthetic lethality in the context of anticancer therapy. Nat Rev Cancer 5: 689-698, 2005.

18. Farmer H, McCabe N, Lord CJ, Tutt AN, Johnson DA, Richardson TB, Santarosa M, Dillon KJ, Hickson I, Knights C, et al: Targeting the DNA repair defect in BRCA mutant cells as a therapeutic strategy. Nature 434: 917-921, 2005.

19. Bryant HE, Schultz N, Thomas HD, Parker KM, Flower D, Lopez E, Kyle S, Meuth M, Curtin NJ and Helleday T: Specific killing of BRCA2-deficient tumours with inhibitors of poly(ADP-ribose) polymerase. Nature 434: 913-917, 2005.

20. Nevanlinna $\mathrm{H}$ and Bartek J: The CHEK2 gene and inherited breast cancer susceptibility. Oncogene 25: 5912-5919, 2006.

21. Bartek J and Lukas J: Chk1 and Chk2 kinases in checkpoint control and cancer. Cancer Cell 3: 421-429, 2003. 